\title{
Identification and validation of the role of matrix metalloproteinase- 1 in cervical cancer
}

\author{
RUN TIAN ${ }^{1}$, XIAOFANG LI $^{2-4}$, YAN'E GAO ${ }^{2}$, YUE LI $^{1}$, PEI YANG ${ }^{1}$ and KUNZHENG WANG ${ }^{1}$ \\ Departments of ${ }^{1}$ Orthopedics and ${ }^{2}$ Obstetrics and Gynecology, The Second Affiliated Hospital, \\ Xi'an Jiaotong University, Xi'an, Shaanxi 710004, P.R. China; ${ }^{3}$ Hudson Institute of Medical Research; \\ ${ }^{4}$ Department of Molecular and Translational Science, Monash University, Clayton, Victoria 3168, Australia
}

Received July 15, 2017; Accepted January 31, 2018

DOI: 10.3892/ijo.2018.4267

\begin{abstract}
Lymph node (LN) metastasis at an early stage of cervical cancer is often an indicator of poor prognosis and is critical for subsequent adjuvant therapy. The current study aimed to identify aberrant gene signatures and biomarkers of metastasis for patients with cervical cancer. RNA-sequencing data of $132 \mathrm{LN}$ negative (N0) and $60 \mathrm{LN}$ positive (N1) cervical cancer samples obtained from The Cancer Genome Atlas database were analyzed. Differentially expressed genes were identified using $\mathrm{R}$ packages 'edgeR' and 'limma'. Kyoto Encyclopedia of Genes and Genomes pathway enrichment and Gene Set Enrichment Analysis (GSEA) were conducted. The GSE9750 dataset obtained from Gene Expression Omnibus was analyzed to identify genes that are persistently aberrantly expressed during the development of cervical cancer. The peroxisome proliferator-activated receptor (PPAR) signaling pathway was screened out to be significant during LN metastasis. In the two analyzed datasets, 11 genes were aberrantly expressed, while matrix metalloproteinase 1 (MMP1) was the only gene that was persistently overexpressed. Cell viability, wound healing and Transwell assays were performed to evaluate the effects of MMP1 knockdown in cervical cancer cell lines, and the expression of epithelial mesenchymal transition (EMT) markers was detected. Finally, the clinical
\end{abstract}

Correspondence to: Professor Kunzheng Wang, Department of Orthopedics, The Second Affiliated Hospital, Xi'an Jiaotong University, 157 Xiwu Road, Xincheng, Xi'an, Shaanxi 710004, P.R. China

E-mail: country__side@126.com

Abbreviations: LN, lymph node; DEGs, differently expressed genes; N0/N1, LN-negative/LN-positive; TCGA, The Cancer Genome Atlas; KEGG, Kyoto Encyclopedia of Genes and Genomes; GSEA, Gene Set Enrichment Analysis; GEO, Gene Expression Omnibus; PPAR, peroxisome proliferator-activated receptor; MMP1, matrix metalloproteinase 1; EMT, epithelial-mesenchymal transition; FIGO, International Federation of Obstetricians and Gynecologists

Key words: cervical cancer, lymph node metastasis, bioinformatics, matrix metalloproteinases-1, epithelial-mesenchymal transition significance of MMP1 was investigated. The current study identified that MMP1 was overexpressed and the PPAR signaling pathway was associated LN metastasis in patients with cervical cancer. Following knockdown of MMP1, the proliferation, migration and invasion of cervical cancer cell lines were weakened, the expression of epithelial marker E-cadherin was increased, and the expression of metastasisassociated gene vimentin was decreased. MMP1 was an independent prognostic factor for cervical cancer. The current study indicated that MMP1 has a key role in the regulation of cervical tumor growth and LN metastasis via EMT to a certain extent. The results suggest that MMP1 may be a biomarker for LN metastasis of cervical cancer, and further validation should be performed.

\section{Introduction}

As the world's second most common type of gynecological cancer, cervical cancer causes $\sim 12,820$ newly diagnosed cases and 4,210 mortalities each year in the United States (1). Although radical surgical treatment and radiotherapy are effective treatments, more than a third of these patients will develop progressive or recurrent tumors $(2,3)$. Currently, the International Federation of Obstetricians and Gynecologists (FIGO) staging system, depth of invasion and lymph node (LN) status are recognized prognosis factors in patients with cervical cancer (4-6). Among these factors, the adverse impact of LN metastasis on patients with cervical cancer was confirmed in certain recent clinical studies $(4,7)$. Currently, there are some studies that have investigated the mechanism of cervical LN metastasis $(8,9)$; however, a there is still no valid biomarker for LN metastasis. Therefore, a comprehensive understanding of the pathways and genes contributing to the development and LN metastasis of cervical cancer is required.

The peroxisome proliferator-activated receptor (PPAR) signaling pathway was first reported in 1990, and demonstrated to be involved in glucose and lipid metabolism (10). With further investigation of the association between cell metabolism and cancer, the role of PPARs in tumors has been continuously explored. In general, deregulation of PPAR $\gamma$, a subtype of PPARs, is detected in peripheral tissues associated with lipid metabolism (11), whereas Mandard and 
Patsouris (12) reported that PPAR $\gamma$ was also deregulated in inflammation and cancer. Although evidence has suggested that PPAR ligands may inhibit tumor angiogenesis during tumor formation $(13,14)$, the importance of PPAR signaling pathway in tumors remains unclear, particularly the role in the carcinogenesis and metastasis of cervical cancer.

As members of the zinc-dependent protease family, matrix metalloproteinases (MMPs) are important factors involved in the degradation of extracellular matrix and proteolysis (15). As an interstitial collagenase, abnormal expression of MMP1 has been reported to be associated with progression of human cancer. The increased expression of MMP1 detected in prostate cancer (16), bladder cancer (17) and gastric cancer (18) has been demonstrated to be closely associated with prognosis. In addition, MMP1 has been reported to promote angiogenesis by activating the endothelial protease-activated receptor-1 (19). Anand et al (20) reported that glioma cell invasion can be promoted by MMP1 through the mitogen-activated protein kinase pathway (20). Whether MMP1 has a role in LN metastasis of cervical cancer is remains unknown.

The Cancer Genome Atlas (TCGA) is a publicly funded project that aims to catalogue and discover major cancercausing genomic alterations. The present study was based on high-throughput RNA-sequencing data from TCGA. Gene profiling, molecular signatures and functional pathway information with Gene Set Enrichment Analysis (GSEA) were incorporated to identify aberrant pathways and genes between patients with cervical cancer that were $\mathrm{LN}$-negative (N0) and LN-positive (N1). The combined approach revealed that MMP1 in the PPAR signaling pathway served a role in cervical cancer LN metastasis. The effect of MMP1 on tumor metastasis phenotype was validated by cervical cancer cell line experiments in vitro. The clinical data in the TCGA database also confirmed the effect of high expression of MMP1 on clinical prognosis. Critically, it was demonstrated that MMP1 had key role in the regulation of metastasis by promoting epithelial-mesenchymal transition (EMT) in cervical cancer and was an independent prognostic factor for cervical cancer. The mechanism of how upregulated MMP1 promotes LN metastasis in cervical cancer requires further validation.

\section{Materials and methods}

Datasets. Transcriptome profiling data and prognostic data of N0 and N1 cervical cancer (portal.gdc.cancer.gov/projects/ TCGA-CESC) were accessed from the TCGA (cancergenome. nih.gov) consortium. In total, 132 N0 and 60 N1 cervical cancer samples were obtained. The gene expression profiles of GSE9750 were downloaded from Gene Expression Omnibus database (ncbi.nlm.nih.gov/geo). GSE9750, which was based on Agilent GPL96 platform (Agilent GeneChip Affymetrix Human Genome U133A Array; Thermo Fisher Scientific, Inc., Waltham, MA, USA), was submitted by Scotto et al (21). The GSE9750 dataset contained 66 samples, including 33 cervical cancer samples, 24 normal cervix epitheliums, and 9 cervical cancer cell lines.

Identification of differentially expressed genes (DEGs). The transcriptome profiling data files obtained from TCGA for analysis were systemized and transferred into a .txt file which included expression and prognosis data using a Perl order line. Then, package 'edgeR' $(22,23)$ of Bioconductor $(24)$ (version 3.4) was applied in RStudio (RStudio, Inc., Boston, MA, USA; version 3.3.2) to screen out the DEGs. The raw gene expression profiles of GSE9750 were read using package 'affyPLM' (23), and probe quality control was determined by relative logarithmic expression. Following preprocessing of the data by log scale robust multi-array analysis and filling the missing values using the k-Nearest Neighbor method, package 'limma' (25) was applied to obtain the DEGs. Exact test was used to search for the non-random association between the expression of genes and group according to the read counts of genes. In order to optimize the probability of random association and the number of DEGs, fold change (FC) $\wedge 2$ and $\mathrm{P}<0.05$ was considered to indicate a statistically significant difference.

Kyoto Encyclopedia of Genes and Genomes (KEGG) pathway enrichment analysis of DEGs. KEGG (kegg.jp) is a knowledge base for systematic and comprehensive analysis of gene functions in pathways and to link genomic information with higher-level function information. Database for Annotation, Visualization and Integrated Discovery (DAVID; david.ncifcrf.gov; version 6.8) is an important foundation for any high-throughput gene functional analysis. ClueGO (version 2.2.3) is a plug-in app of Cytoscape (cytoscape.org; version 3.5.0) where KEGG pathway enrichment analysis can also be performed based on a different database from DAVID. In order to analyze the DEGs at the functional level, KEGG pathway analysis was applied by ClueGo and verified by DAVID. $\mathrm{P}<0.05$ was considered to indicate statistical significance, pathways including four or more DEGs are presented in the ClueGo-KEGG figures.

Pathway gene signatures analyzed using GSEA. GSEA (version 6.0) $(26,27)$ is a computational method for exploring whether a given gene set is significantly enriched in a group of gene markers ranked by their relevance with a phenotype of interest. The curated KEGG pathway V6.0 dataset was used to compare the impaired pathways in $\mathrm{N} 0$ and $\mathrm{N} 1$ cervical cancer samples. Additionally, the gene sets of $<15$ genes or $>500$ genes were excluded. The phenotype label was set as N1 vs. N0. The t-statistic mean of the genes was computed in each KEGG pathway using a permutation test with 1,000 replications. The upregulated pathways were defined by a normalized enrichment score (NES) $>0$ and the downregulated pathways were defined by an NES $<0$. Pathways with a false discovery rate $\mathrm{P} \leq 0.1$ were considered significantly enriched.

Cell culture. Human cervical cancer cell lines HeLa and SiHa were purchased from (American Type Culture Collection, Manassas, VA, USA). Cells were cultured in Dulbecco's modified Eagle's medium (Sigma-Aldrich; Merck KGaA, Darmstadt, Germany) supplemented with $10 \%$ fetal bovine serum (FBS; Gibco; Thermo Fisher Scientific, Inc.) at $37^{\circ} \mathrm{C}$ with $5 \% \mathrm{CO}_{2}$ in a cell culture incubator.

Knockdown of MMPl. For designing the single guide RNA (sgRNA), MIT Clustered Regularly Interspaced Short Palindromic Repeats (CRISPR) design software was used (crispr.mit.edu). The MMP1 sgRNA sequence was as follows: 
TCACTGAGGGGAACCCTCGC (exon 2; score, 89). The lentiviral particles were produced by transient transfection of letiX-293 cells cultured in a 10-cm Petri dishes with $10 \mu \mathrm{g}$ vector DNA pFgh1tUTG (a gift from Dr Marco Herold; plasmid no. 70183, Addgene, Inc., Cambridge, MA, USA) (28) with the target sgRNA $(10 \mu \mathrm{g})$ inserted and the pFUCas9mCherry vector $(10 \mu \mathrm{g})$, along with the packaging constructs pMDL $(5 \mu \mathrm{g})$, pRSV-rev $(2.5 \mu \mathrm{g})$, and pVSV-G $(3 \mu \mathrm{g}$; gifts from Dr Dakang Xu; Hudson Institute of Medical Research) using standard calcium phosphate precipitation method. Virus-containing supernatants were collected at $48 \mathrm{~h}$ after transfection and passed through a $0.45 \mu \mathrm{m}$ filter. To establish MMP1 knockdown cervical cancer cells, HeLa and SiHa cells were treated with $8 \mathrm{ng} / \mathrm{ml}$ polybrene in the viral supernatant, incubated for $30 \mathrm{~min}$ at $37^{\circ} \mathrm{C}$, and then centrifuged at $500 \mathrm{x} \mathrm{g}$ for $2 \mathrm{~h}$ at $32^{\circ} \mathrm{C}$. Doxycycline hyclate $(1 \mu \mathrm{g} / \mathrm{ml}$; Sigma-Aldrich; Merck KGaA) was used for treatment of cell lines to induce expression of the sgRNA for 3 days. Cells without doxycycline hyclate treatment were used as the knockdown control. Western blotting was performed to assess the efficacy of the knockdown.

Western blotting. Cells were washed twice with cold PBS, followed by lysis buffer [Tris-HCl (3.03 g), SDS (0.5 g), $\mathrm{NaCl}(4.35 \mathrm{~g}), \mathrm{NaN}_{3}(0.1 \mathrm{~g})$, deoxysodium cholate $(2.5 \mathrm{~g})$, $1 \% \mathrm{NP}-40(5 \mathrm{ml})$ in $11 \mathrm{ddH}_{2} \mathrm{O}$, plus $100 \mathrm{mM}$ phenylmethane sulfonyl fluoride (1:100) and inhibitor cocktail (1:50; cat. no. P8340; Sigma-Aldrich; Merck KGaA) added before use]. The concentration of the protein was determined by bicinchoninic acid protein assay kit (Merck KGaA). Protein lysate (2 $\mu \mathrm{g}$ per well) was separated on $10 \%$ SDS-PAGE gels and transferred onto nitrocellulose membranes (PerkinElmer, Inc., Waltham, MA, USA) using standard western blotting protocols. After blocking in Odyssey blocking buffer (cat. no. 927-40000; LI-COR Biosciences, Lincoln, NE, USA) for 50 min, primary antibody against MMP1 (1:500 dilution; cat. no. sc-21731; Santa Cruz Biotechnology, Inc., Dallas, TX, USA), $\beta$-actin (1:10,000 dilution; cat. no. ab8227; Abcam, Cambridge, MA, USA), E-cadherin (1:1,000 dilution; cat. no. 3195; Cell Signaling Technology, Inc., Danvers, MA, USA) and vimentin (1:1,000 dilution; cat. no. 5741; Cell Signaling Technology, Inc.) were incubated overnight at $4^{\circ} \mathrm{C}$ and the proteins were detected using secondary antibodies conjugated to IRdye680 (cat. no. 18-4416-32/18-4417-32; Rockland Immunochemicals Inc., Limerick, PA, USA) or IRdye800 (cat. no. 18-4516-32/18-4517-32; Rockland Immunochemicals Inc.) were used to image the proteins at 700 or $800 \mathrm{~nm}$ under a LI-COR-Odyssey imaging system (LI-COR Biosciences) scanner.

Proliferation assay. Cell viability was measured over a period of 6 days using the PrestoBlue kit (Invitrogen; Thermo Fisher Scientific, Inc.). Cells in the log growth phase were seeded into a 96-well plate $(2,000$ cells/wells) and adhered overnight. Following the manufacturer's instructions, $10 \mu \mathrm{l}$ PrestoBlue reagent and $90 \mu \mathrm{l}$ fresh medium were added to each well. After $30 \mathrm{~min}$ incubation at $37^{\circ} \mathrm{C}$, the fluorescence at a wavelength of $570 \mathrm{~nm}$ was read using a FLUOstar Optima microplate spectrophotometer from BMG Labtech GmbH (Ortenberg, Germany). The reagent was replaced with fresh medium, and repeated on the next day. All experiments were performed three times independently in biological triplicate.

Migration and invasion assays. Cell migration activity was assessed by a wound healing assay. Briefly, $10^{6}$ cells were seeded into 6-well plate at $90 \%$ confluency and adhered overnight. The cells were wounded by scratching lines with a 200- $\mu 1$ pipette tip. Floating cells were washed off with 1X PBS, then the cells were observed and imaged under a microscope at different time-points. The distance of the uncovered wound gap was quantified using ImageJ software (version: 1.8.0; National Institutes of Health, Bethesda, MD, USA), the relative wound closure was assessed at $36 \mathrm{~h}$ relative to $0 \mathrm{~h}$. A cell invasion assay was performed using Transwell chambers (24-well, $8 \mu \mathrm{m}$ pore size; Corning Life Sciences, Corning, NJ, USA). In this assay, $600 \mu 1$ RPMI medium containing $25 \%$ FBS was used as the attractant in the lower chamber, and $100 \mu 1$ RPMI medium containing 5\% FBS with $10^{5}$ cells was added to the upper chamber; the cells were allowed to migrate through the pores for $36 \mathrm{~h}$. The cells on the lower side of the filter were fixed with $4 \%$ paraformaldehyde for $10 \mathrm{~min}$ and were stained with $0.5 \%$ crystal violet for $15 \mathrm{~min}$ at room temperature. The number of the cells on the underside of the filter from three randomly selected microscopic views were counted and digitally imaged. All experiments were performed three times independently in biological triplicate.

$R N A$ isolation and reverse transcription-quantitative polymerase chain reaction ( $R T-q P C R)$. Total RNA was extracted from cells using TRIzol (Invitrogen; Thermo Fisher Scientific, Inc.) according to the manufacturer's protocol. RT of cDNA was synthesized using the Thermo Scientific RevertAid First Strand cDNA Synthesis kit (cat. no. K1622; Thermo Fisher Scientific, Inc.) according to the manufacturer's instructions. The mixture of template and primer was incubated at $65^{\circ} \mathrm{C}$ for $5 \mathrm{~min}$, then the following substances were added in sequence and incubated at $42^{\circ} \mathrm{C}$ for $30 \mathrm{~min}$ : Reaction buffer, $4 \mu \mathrm{l}$; RiboLock $^{\mathrm{TM}}$ RNA, $1 \mu 1$; dNTP mix, $2 \mu \mathrm{l}$; RevertAid ${ }^{\mathrm{TM}}$ $\mathrm{M}-\mathrm{MuLV}, 1 \mu \mathrm{l}$. The reaction was terminated by $70^{\circ} \mathrm{C}$ for $5 \mathrm{~min}$. The mRNA levels were determined using Maxima ${ }^{\mathrm{TM}}$ SYBR Green/ROX qPCR Master Mix (2X; cat. no. K0222 Thermo Fisher Scientific, Inc.) on ABI 7500 Real-Time PCR Systems. The PCR conditions were as follows: Preheating at $50^{\circ} \mathrm{C}$ for $2 \mathrm{~min}$; initial denaturation at $95^{\circ} \mathrm{C}$ for $10 \mathrm{~min} ; 40$ denaturation cycles at $95^{\circ} \mathrm{C}$ for $15 \mathrm{sec}$; and primer annealing/elongation at $60^{\circ} \mathrm{C}$ for $1 \mathrm{~min}$. Melting curves were analyzed in each run to confirm specificity of amplification. 18S rRNA were used as internal normalization controls. Primers specific to E-cadherin and vimentin were as follows: E-cadherin, forward 5'-GAA CGCATTGCCACATACAC-3', reverse 5'-AGCACCTTCCAT GACAGACC-3'; vimentin, forward 5'-CCCTCACCTGTGAA GTGGAT-3', reverse 5'-GACGAGCCATTTCCTCCTTC-3'; and $18 \mathrm{~S}$ rRNA, forward 5'-CAAGCCCGACTTTGCAGA-3', reverse 5'-CGCACGATTCGTCAAGTTATC-3'. The $2^{-\Delta \Delta C q}$ method was used to represent fold change $(29,30)$. In each experiment, E-cadherin/18S and vimentin/18S levels in MMP1 knockdown cells were normalized to E-cadherin/18S and vimentin/18S levels in MMP1-expressing cells. All experiments were performed in biological triplicate. Data are presented as the mean \pm standard error of the mean (SEM). 
A

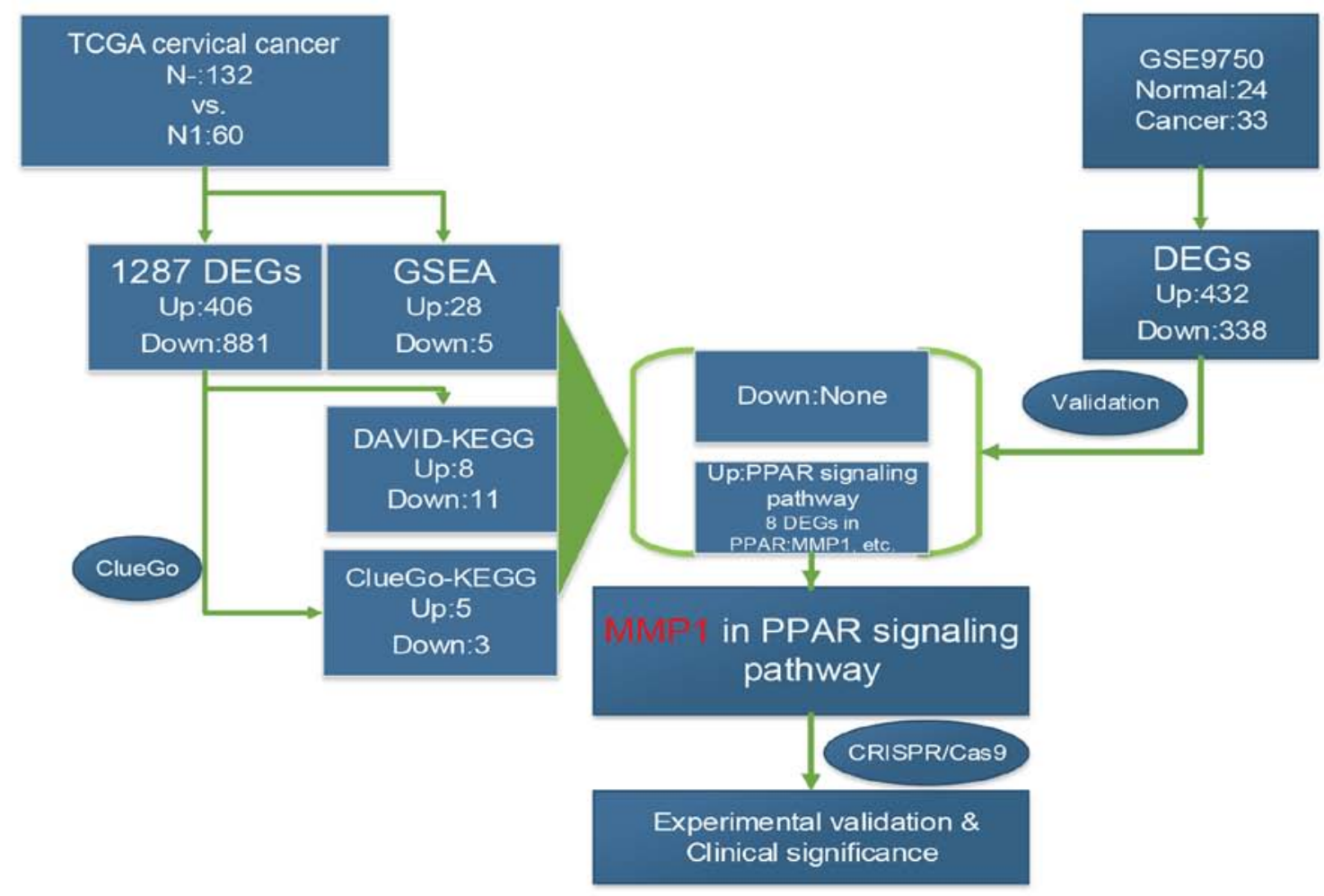

B

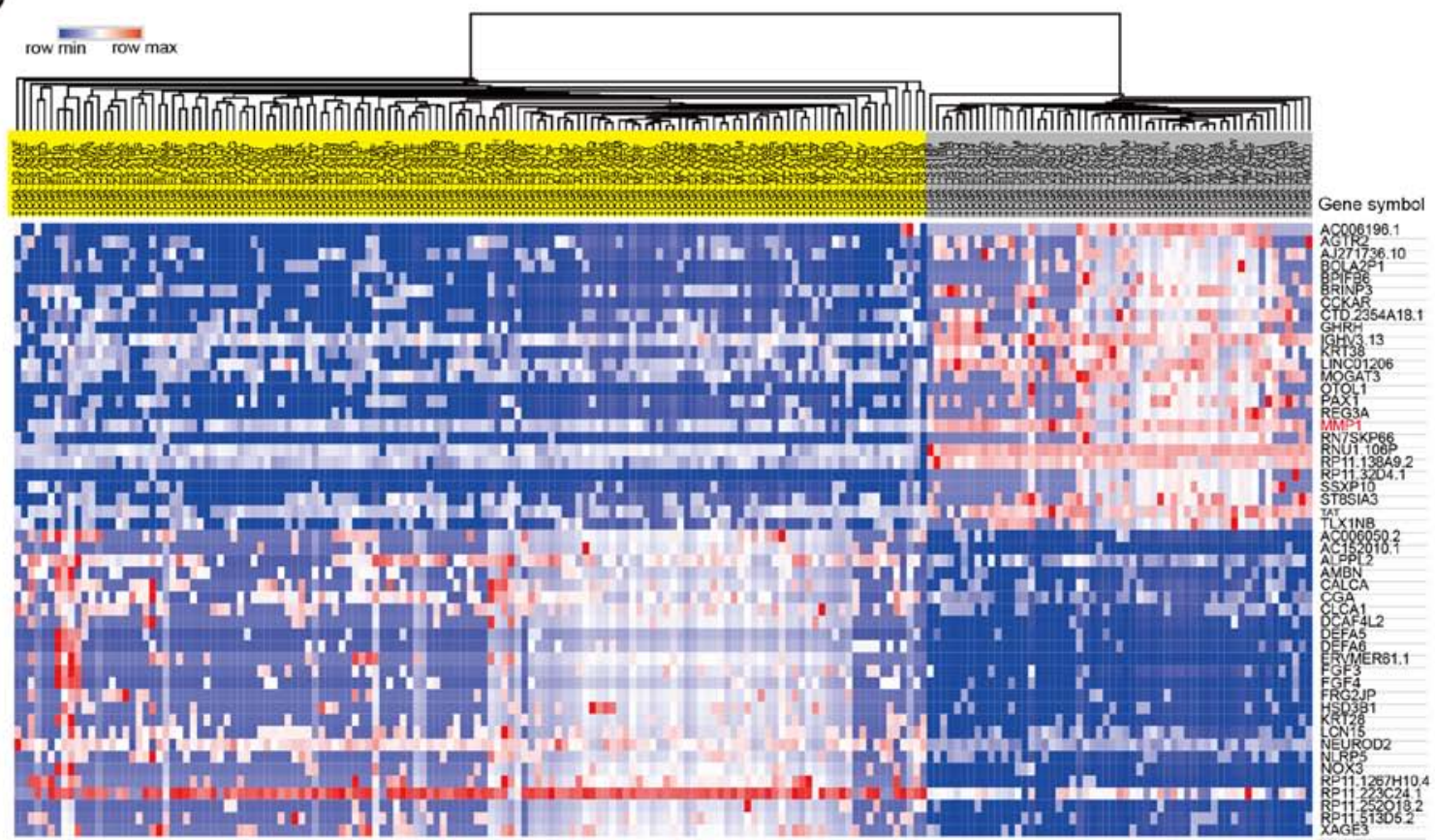

Figure 1. Work flow and heatmap of the top 25 DEGs. (A) Work-flow of the current study. (B) Top 25 up- and downregulated DEGs in a heatmap. The upper section represents the hierarchical clustering results, with yellow and grey bars indicating N0 and N1 samples respectively. Genes are listed on the right and red grids represented high expression while blue grids represented low expression in N1 vs. N0. TCGA, The Cancer Genome Atlas; DEG, differentially expressed gene; GSEA, Gene Set Enrichment Analysis; DAVID, Database for Annotation, Visualization and Integrated Discovery; KEGG, Kyoto Encyclopedia of Genes and Genomes; PPAR, peroxisome proliferator-activated receptor; MMP1, matrix metalloproteinase 1; CRISPR, Clustered Regularly Interspaced Short Palindromic Repeats.

Survival analysis of N1 vs. N0 cervical cancer patients and DEGs. Survival analysis of N1 vs. N0 cervical cancer patients and DEGs in overlapped pathway was conducted.
Package 'survival' (version 2.38; CRAN.R-project.org/ package $=$ survival) in RStudio was applied and Kaplan-Meier curves were mapped based on the follow-up data from TCGA. 
A

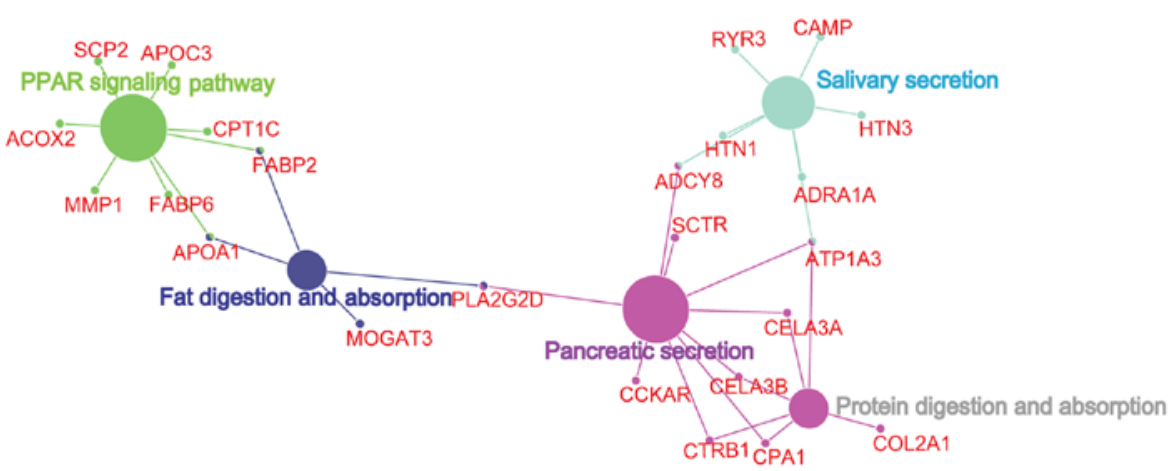

B

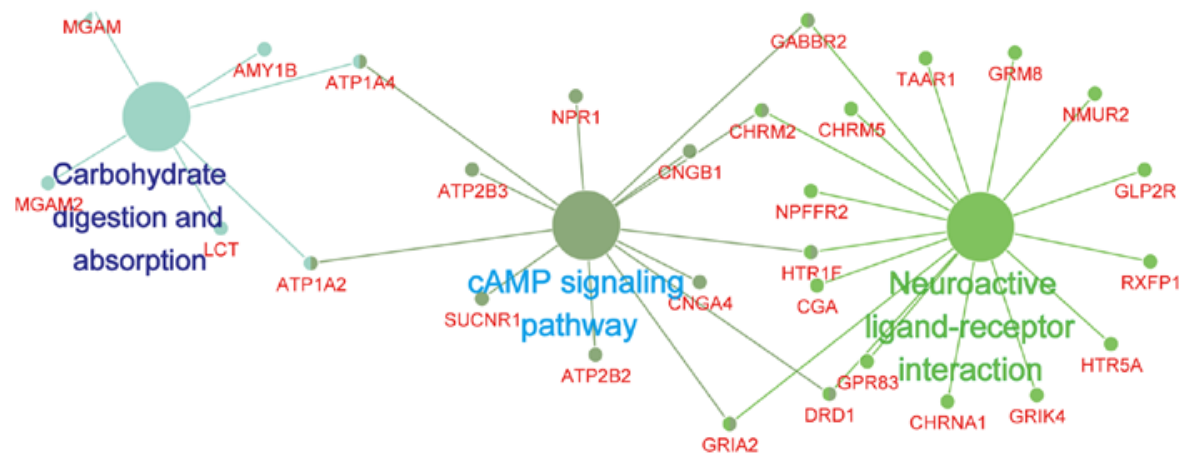

Figure 2. Identification of upregulated pathways based on ClueGO. (A) Significantly upregulated pathways of upregulated DEGs between N0 and N1 based on ClueGO. (B) Significantly downregulated pathways of downregulated DEGs between N0 and N1 based on ClueGO. Large circles are pathways and small circles indicate genes in the pathways. If the genes were part of more than two pathways, it is indicated as a fusion of the colors from the two pathways. $\mathrm{P}<0.05$ was considered to indicate a statistically significant difference, pathways including four or more DEGs are presented in ClueGo-Kyoto Encyclopedia of Genes and Genomes figures. DEGs, differentially expressed genes; PPAR, peroxisome proliferator-activated receptor; cAMP, cyclic adenosine monophosphate.

A log-rank test was used to calculate the statistical significance of the difference in survival between the two groups and the cut-off of $\mathrm{P}<0.05$ was consider to be a statistically significant difference.

Statistical analysis. Statistical analysis was performed using GraphPad Prism software (version 7.0; GraphPad Software, Inc., La Jolla, CA, USA) and displayed as the mean \pm SEM. SPSS (version 22.0; IBM Corp., Armonk, NY, USA) was utilized to perform receiver operating characteristic (ROC) curve analysis and Cox's proportional hazards regression model. A significant difference between two groups was analyzed using unpaired Student's t-test with Welch's correction. Survival curves were constructed with the Kaplan-Meier method and compared using the log-rank test. $\mathrm{P}<0.05$ was considered to indicate a statistically significant difference.

\section{Results}

Work flow for the identification of key pathways and genes in LN metastasis of cervical cancer. N1 and N0 cervical cancer samples were compared to identify significant signatures in cervical cancer (Fig. 1A). In total, 1,287 DEGs, including 406 upregulated and 881 downregulated genes (N1 vs. N0), were obtained based on 132 N0 and 60 N1 cervical cancer samples from TCGA. The top 25 of up and downregulated DEGs are presented in Fig. 1B. PPAR signaling pathway was identified to be the top distinct signature upregulated pathway by different enrichment methods (KEGG pathway enrichment and GSAE). To explore further, GSE9750 datasets were analyzed and 770 DEGs were obtained. There were 11 mutual DEGs selected following overlapping DEGs from two different cohorts. Among 11 mutual DEGs, MMP1 in PPAR signaling pathway was the unique gene with abnormal high expression. Finally, two cervical cell lines were chosen to verify the significance of MMP1 on the invasion and metastasis, and clinical significance of MMP1 was investigated (Fig. 1).

Identification of distinct pathways for in cervical cancer. Up and downregulated DEGs between N0 and N1 were analyzed to determine the most significantly enriched pathways based on ClueGO and DAVID. The ClueGO result demonstrated that 'PPAR signaling pathway', 'Fat digestion and absorption', 'Protein digestion and absorption' and others were significantly enriched (Fig. 2A), while the most significant pathways enriched in the downregulated DEGs were 'Carbohydrate digestion and absorption', 'cAMP signaling pathway' and 'Neuroactive ligand-receptor interaction' based on the ClueGO database (Fig. 2B). All the five upregulated and three downregulated pathways enriched by ClueGO were validated by DAVID.

GSEA was conducted to investigate the differential pathways between N0 and N1. Finally, 28 upregulated and 5 downregulated pathways were identified (data not shown). 

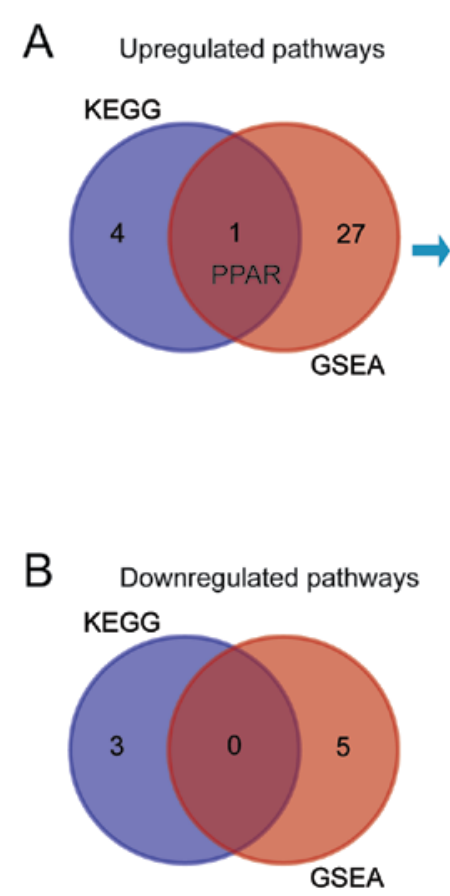

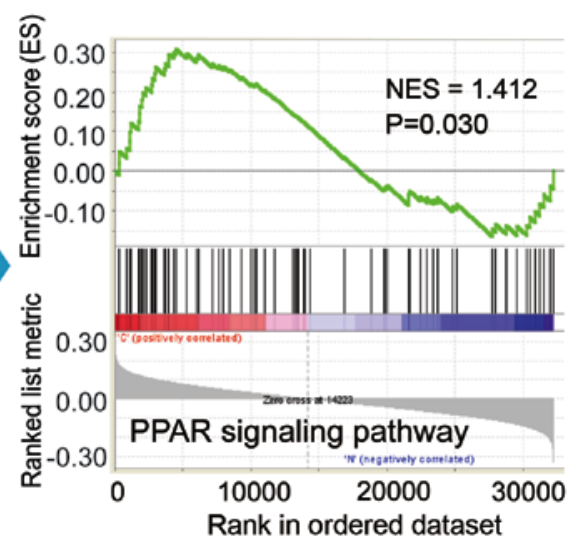

C Overlapped DEGs

Normal vs. cancer

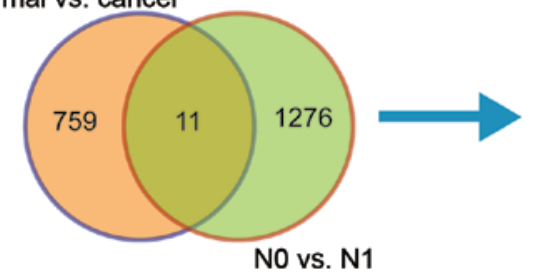

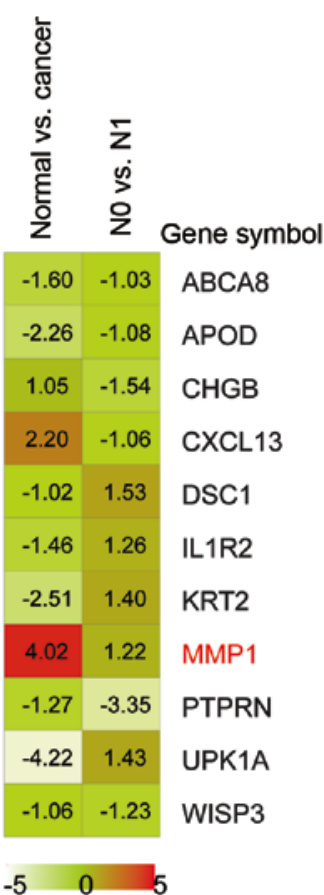

Figure 3. Identification of key gene signatures and key genes in cervical cancer. (A) Venn diagram illustrating the overlapped pathways among KEGG and GSEA (left) and the representative GSEA curve of mutual upregulated pathway PPAR signaling (right) with NES=1.413 and P=0.030 when comparing N1 and N0. (B) Venn diagram illustrating the overlapped pathways between KEGG and GSEA for downregulated pathways. (C) Venn-diagram illustrating the overlapped DEGs between another GEO cohort (normal vs. cancer, GSE 9750; N0 vs. N1, TCGA; left) and a heatmap illustrating the log fold change of the 11 overlapped genes in the two groups (right). MMP1 is marked as red for its elevation in both groups. KEGG, Kyoto Encyclopedia of Genes and Genomes; PPAR, peroxisome proliferator-activated receptor; GSEA, Gene Set Enrichment Analysis; NES, normalized enrichment score; DEGs, differentially expressed genes; ABCA8, ATP binding cassette subfamily A member 8; APOD, apolipoprotein D; CHGB, chromogranin B; CXCL13, C-X-C motif chemokine ligand 13; DSC1, desmocollin 1; IL1R2, interleukin 1 receptor type 2; KRT2, keratin 2; MMP1, matrix metalloproteinase 1; PTPRN, protein tyrosine phosphatase, receptor type N; UPK1A, uroplakin 1A; WISP3, WNT1 inducible signaling pathway protein 3.

Identification of key gene signatures in cervical cancer. Overlapping the GSEA results with the KEGG pathways produced one upregulated pathway, namely 'PPAR signaling pathway' and no downregulated pathways were mutual in KEGG and GSEA. The peak in the GSEA result of the PPAR signaling pathway was shifted left, with NES=1.412, indicating that most of the genes in PPAR signaling pathway were upregulated (Fig. 3A and B). Fig. 3C There were 11 mutual genes in the N0 vs. N1 cohort (1,287 DEGs) and normal vs. cancer cohort (770 DEGs). Among the 11 mutual genes, MMP1 in the 'PPAR signaling pathway' was the only gene with high expression in cervical cancer vs. normal cervix and $\mathrm{N} 1$ vs. N0 cervical cancer ( $\log \mathrm{FC} 4.02$ and 1.22, respectively).

Knockdown of MMP1 alters malignant behavior in vitro. Cell proliferation, migration and invasion have important roles in the carcinogenesis. Different functional assays were performed to explore the functional significance of MMP1. Inducible CRISPR gene editing of MMP1 was conducted, and the knockdown efficiency was displayed by immunoblotting (Fig. 4A). To determine the contribution of MMP1 in cervical cancer cell growth, cell viability was determined. Growth of MMP1 knockdown cells was significantly reduced compared with the control cells by day 5 and 6 , while there was no significant difference between MMP1 knockdown cells and control cells before day 4 (Fig. 4B). Cell motility was determined by wound healing assay and Transwell assay. The migration of MMP1 knockdown cell lines was assessed by measuring the scratch wound closure capacity. There was a significant delay in wound closure in the MMP1 knockdown cells at $36 \mathrm{~h}$ compared with the control cells $(\mathrm{P}<0.05$; percentage of wound closure) with $70-80 \%$ of the wound remaining open; in control cells, $\sim 40 \%$ of the wound remained open (Fig. 4C). The Transwell assay demonstrated that $\sim 20$ cells per field were observed in MMP1 knockdown cells, and the control cells had 70-80 cells per field, which demonstrated the reduced invasion ability in MMP1 knockdown cells compared with the control $(\mathrm{P}<0.05$; Fig. 4D). Taken together, the data suggested that knockdown of MMP1 decreased the proliferation, invasion and migration ability in SiHa and HeLa cervical cancer cells lines.

Knockdown of MMP1 inhibited EMT of cervical cancer cells. To explore the mechanism of MMP1 in promoting metastasis, EMT markers were detected. The expression of epithelial marker, E-cadherin, was increased significantly $(\mathrm{P}<0.05)$, while the metastasis-associated gene, vimentin, was decreased significantly $(\mathrm{P}<0.05)$ in MMP1 knockdown cell lines comparing with control cervical cancer cell lines (Fig. 5A and B). The results suggested that knockdown of MMP1 may 
A

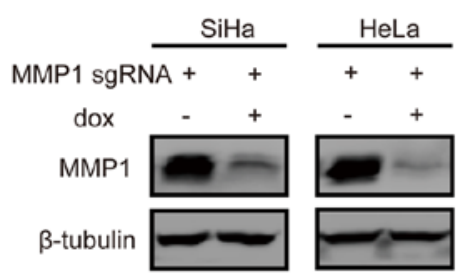

C
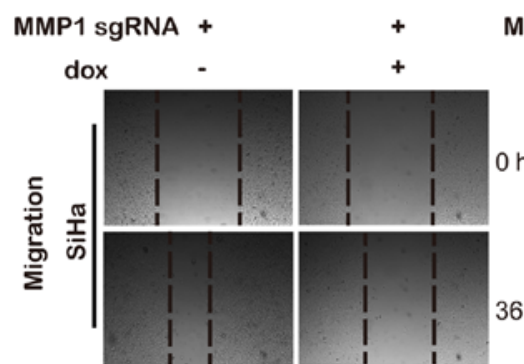

D

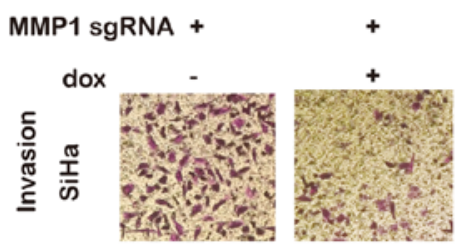

MMP1 sgRNA +

dox
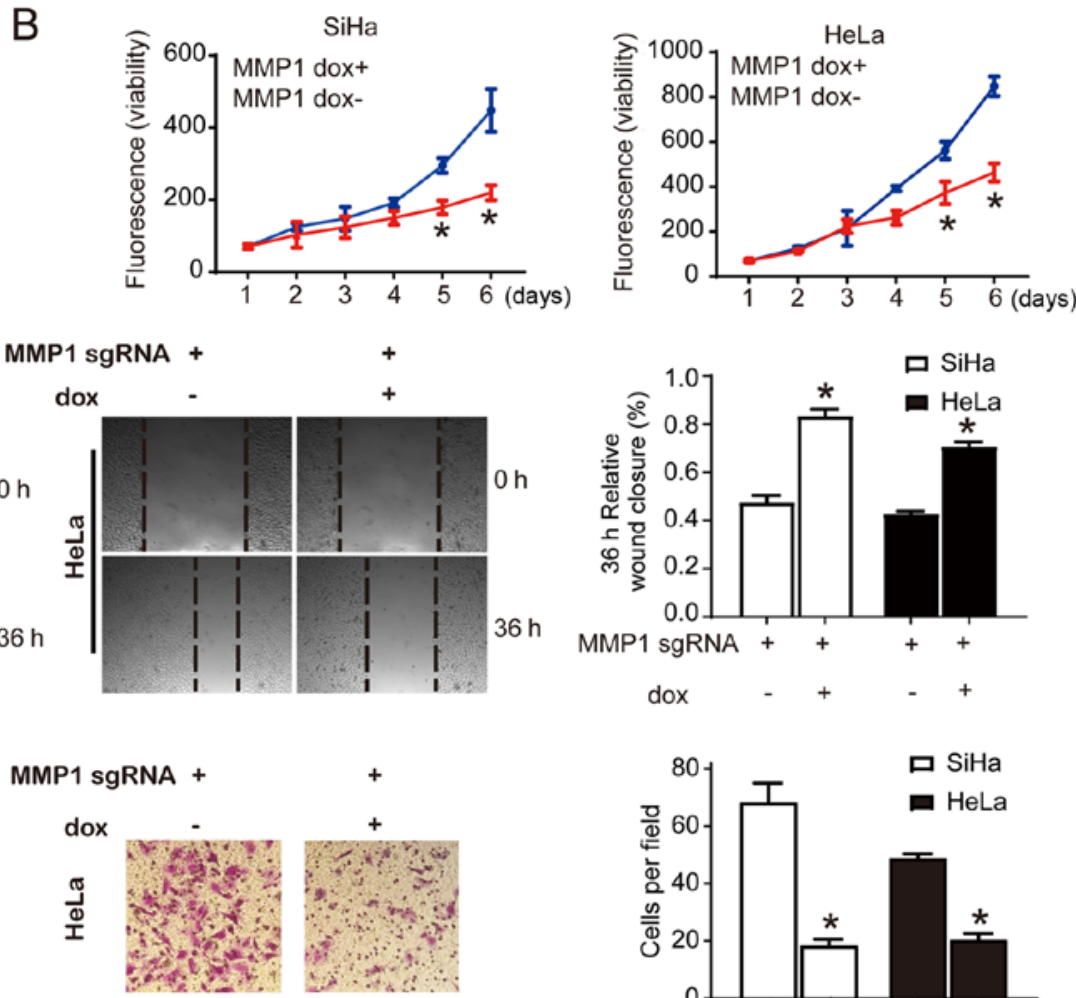

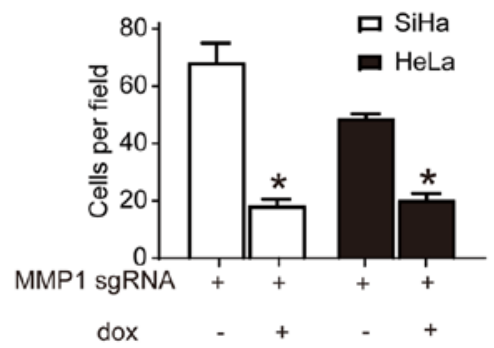

Figure 4. Knockdown of MMP1 alters malignant behavior in vitro. (A) Efficacy of MMP1 CRISPR was demonstrated by western blotting. (B) Cell viability of MMP1 knockdown and control cells was detected by PrestoBlue, relative growth was represented by fluorescence at a wavelength of $570 \mathrm{~nm}$. MMP1 knockdown cells grew more slowly on day 5 and 6 compared with control cells. (C) Cell motility was analyzed by wound healing assay, bar chart displayed the $36 \mathrm{~h}$ relative wound closure of SiHa and HeLa MMP1 knockdown and control cells. (D) Cell invasion was analyzed Transwell assay, images at $36 \mathrm{~h}$ are presented and bar chart displays the cell count per field from random three fields. Data are presented as the mean \pm standard error ( $n=3$; " $\mathrm{P} \leq 0.05$ vs. dox-). MMP1, matrix metalloproteinase 1; sgRNA, single guide RNA; dox, doxycycline hyclate.

A

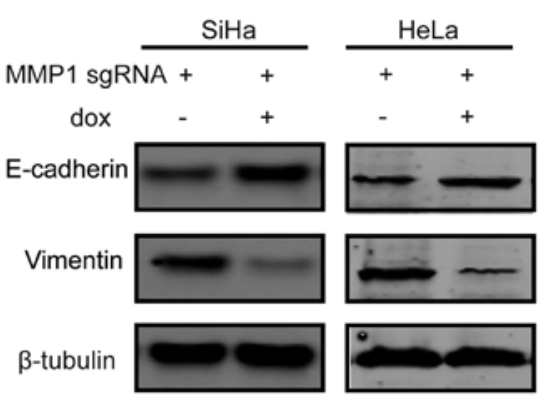

B

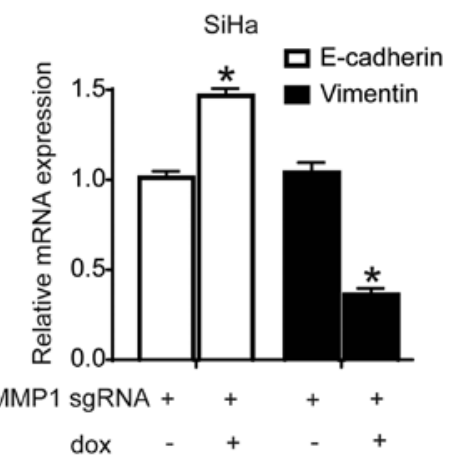

HeLa

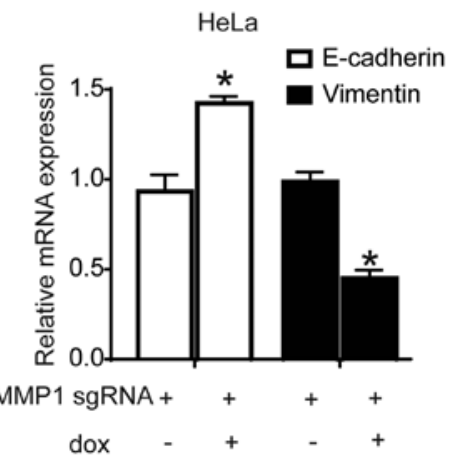

Figure 5. Effect of MMP1 knockdown on epithelial-mesenchymal transition markers. (A) Protein expression of E-cadherin and vimentin as detected by western blotting in each group. The expression of E-cadherin was increased and vimentin was decreased in MMP1 knockdown cervical cancer cell lines. (B) Relative mRNA expression of E-cadherin and vimentin were detected by reverse transcription-quantitative polymerase chain reaction. "P<0.05 vs. dox-. MMP1, matrix metalloproteinase 1; sgRNA, single guide RNA; dox, doxycycline hyclate. 
A

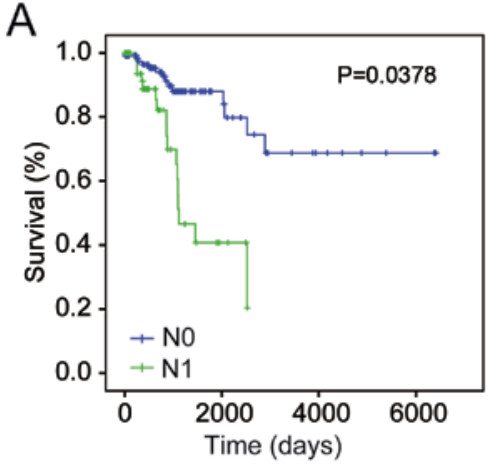

C

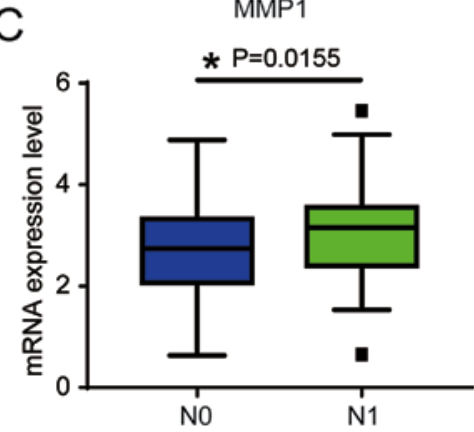

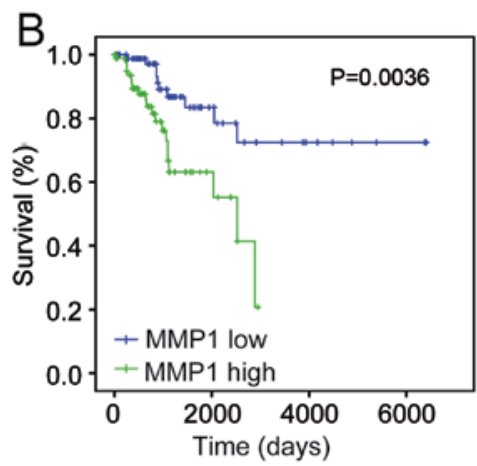

MMP1 (N0 vs. N1)

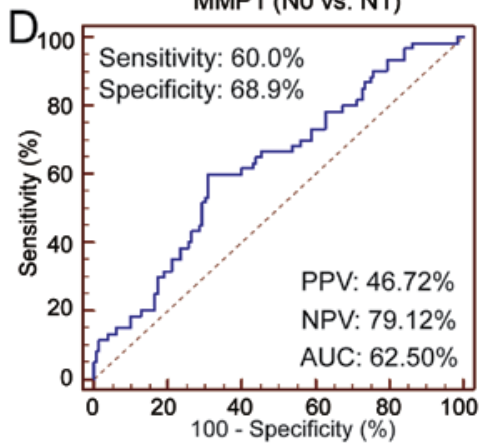

Figure 6. Clinical significance of MMP1 in cervical cancer. (A) The survival curve of patients with N0 and N1 cervical cancer based on The Cancer Genome Atlas demonstrates that $\mathrm{N} 1$ patients had poor prognosis $(\mathrm{P}=0.03789)$. (B) Expression level of MMP1 are significantly associated with the overall survival of patients with cervical cancer and highly expressed MMP1 patients had a poor prognosis $(\mathrm{P}=0.0036)$. (C) MMP1 expression was significantly increased in samples from N1 compared with that in N0 (P=0.0434). (D) A receiver operating characteristic curve of MMP1 was shown for discriminating between N0 and N1. The sensitivity and specificity of MMP1 were $>60 \%$ (60.00\% and $68.90 \%$, respectively). N0, lymph node-negative; N1, lymph node-positive; MMP1, matrix metalloproteinase 1; PPV, positive predictive value; NPV, negative predictive value; AUC, area under curve.

Table I. Univariate and multivariate Cox proportional hazards analysis of MMP1 expression and overall survival of patients with cervical cancer in The Cancer Genome Atlas cohort.

\begin{tabular}{|c|c|c|c|c|}
\hline \multirow[t]{2}{*}{ Factor } & \multicolumn{2}{|c|}{ Univariate analysis } & \multicolumn{2}{|c|}{ Multivariate analysis } \\
\hline & $\mathrm{HR}(95 \% \mathrm{CI})$ & P-value & $\operatorname{HR}(95 \% \mathrm{CI})$ & P-value \\
\hline Age & $0.992(0.978-1.006)$ & 0.272 & & \\
\hline \multicolumn{5}{|l|}{ Stage } \\
\hline I-IIA vs. IIB-IV & $1.536(1.040-2.269)$ & $0.031^{\mathrm{a}}$ & $1.451(1.028-2.413)$ & $0.040^{\mathrm{a}}$ \\
\hline \multicolumn{5}{|l|}{ Grade } \\
\hline $\mathrm{G} 1$ vs. $\mathrm{G} 2+\mathrm{G} 3+\mathrm{G} 4$ & $1.304(0.940-1.810)$ & 0.112 & & \\
\hline \multicolumn{5}{|l|}{ Lymph node } \\
\hline N0 vs. N1 & $3.158(1.544-6.458)$ & $0.002^{\mathrm{b}}$ & $2.560(1.177-5.568)$ & $0.018^{\mathrm{a}}$ \\
\hline \multicolumn{5}{|l|}{$\mathrm{T}$} \\
\hline $\mathrm{T} 1$ vs. $\mathrm{T} 2+\mathrm{T} 3+\mathrm{T} 4$ & $1.015(0.452-2.277)$ & 0.972 & & \\
\hline \multicolumn{5}{|l|}{ MMP1 } \\
\hline High vs. low & $2.014(1.357-2.989)$ & $0.001^{\mathrm{a}}$ & $1.699(1.133-2.548)$ & $0.010^{\mathrm{a}}$ \\
\hline
\end{tabular}

${ }^{\mathrm{a}} \mathrm{P}<0.05,{ }^{\mathrm{b}} \mathrm{P}<0.01$. MMP1, matrix metalloproteinase 1; HR, hazard ratio; CI, confidence interval; N0, lymph node-negative; N1, lymph node-positive.

decrease cell migration and invasion ability by inhibiting EMT in cervical cancer cells.

Clinical significance of MMP1 in patients with cervical cancer. Kaplan-Meier curve analysis TCGA data from patients with N0 and N1 cervical cancer demonstrated that $\mathrm{N} 1$ patients had poor prognosis $(\mathrm{P}=0.03789)$ compared with No patients, which demonstrated the crucial clinical value of predicting LN metastasis (Fig. 6A). The expression level of MMP1 was divided into high expression group and low 
expression group according to the median expression, and was significantly associated the overall survival of patients with cervical cancer, and patients with MMP1 high expression had poor prognosis ( $\mathrm{P}=0.0036$; Fig. 6B). Additionally, MMP1 expression was significantly increased in $\mathrm{N} 1$ cervical cancer samples compared with that in N0 ( $\mathrm{P}=0.0434$; Fig. 6C). The performance of MMP1 for discriminating between N0 and N1 in patients with cervical cancer was determined using an ROC curve. The sensitivity and specificity of MMP1 were $>60 \%$ (60.00\% and 68.90\%, respectively; Fig. 6D). Cox multivariate analysis indicated that FIGO stage [hazard ratio $(\mathrm{HR})=1.451$, $95 \%$ confidence interval $(\mathrm{CI}) 1.028-2.413, \mathrm{P}=0.040), \mathrm{LN}$ status $(\mathrm{HR}=2.560,95 \%$ CI 1.177-5.568, $\mathrm{P}=0.018)$ and MMP1 $(\mathrm{HR}=1.699,95 \%$ CI 1.133-2.548, $\mathrm{P}=0.010)$ were significant independent favorable prognostic factors of OS for patients with cervical cancer (Table I).

\section{Discussion}

$\mathrm{LN}$ metastasis is considered as one of the strongest prognostic markers for patients with cervical cancer; however, the molecular mechanisms involved remain unclear. In the current study, integrative bioinformatics methods and databases identified that the PPAR signaling pathway and MMP1 were vital aberrant signatures during LN metastasis of cervical cancer.

Currently, the association between metabolism and cancer is attracting more and more attention. The PPAR signaling pathway is predominantly involved in lipid and glucose metabolism. Michalik et al (31) reported that the increase in $\operatorname{PPAR} \beta / \delta$ expression was associated with a decrease in lipid accumulation in cardiac cells, whereas overexpression in the intestine was associated with the development of colon cancer. Disordered metabolism will further lead to producing high quantities of inflammatory cytokines, predominantly leptin, and also interleukin (IL)-8, IL-6 and angiopoietin like 4 (32-34). IL-6, a known upstream activator of signal transducer and activator of transcription 3 (STAT3), has been demonstrated to be crucial in the metastasis of various types of human cancer $(35,36)$. Additionally, other studies have also directly confirmed the association between PPARs and tumors. Trombetta et al (37) reported that activation of PPAR $\gamma$ in cancer cells affected tumor development. PPAR $\gamma$ ligand may be effective in inhibiting tumor angiogenesis $(13,38)$, and was recognized as important in the carcinogenesis and metastasis of tumors. In the present study, PPAR signaling was identified as an essential pathway in LN metastasis based on a cohort containing 192 patients with cervical cancer. MMP1 in the PPAR signaling pathway was a DEG with increased expression in the cancer vs. normal and the N1 vs. N0 datasets. Considering the anomalies of inflammatory factors and tumor microenvironment caused by aberrant PPARs and MMP1, it requires further investigation to understand the crucial role of the PPAR signaling pathway and MMP1 during the development of cervical cancer and LN metastasis.

To further validate the role of MMP1 in cervical cancer, CRISPR/Cas9 genome editing was performed to knockdown MMP1 in SiHa and HeLa cervical cancer cell lines and revealed that cell proliferation, migration and invasion abilities were reduced significantly by MMP1 knockdown. Mechanistic studies demonstrated that knockdown of MMP1 may decrease cell invasion and migration ability by inhibiting the EMT process. MMP1 has been reported to have an important role in various types of human tumor. George et al (39) detected abnormal expression of MMP1 in oral squamous cell carcinoma with high histopathological grade. Langenskiöld et al (40) demonstrated that MMP1 was an independent prognostic factor for colon cancer. Currently, certain studies suggested that MMP1 promoted tumor cell migration by degrading specific substrates that regulate cell adhesion and cell-matrix adhesion (41), and the subtle balance between MMPs and tissue inhibitor of metalloproteinases contributed to the tumor microenvironment (42), which is one of the most important aspects of tumor invasion and metastasis (43). MMP1 was reported to be involved in tumor LN metastasis (44), and also to facilitate peritoneal dissemination (45) and nerve tract migration (46). The results of the current study suggested that knockdown of MMP1 decreased the invasion and migration ability of SiHa and HeLa cell lines, which partially supports by the findings of Sun et al (47). Furthermore, the present study demonstrated that knockdown of MMP1 inhibited EMT in SiHa and HeLa cell lines, suggesting that MMP1 may manifest its malignant ability via EMT. Currently, there are several lines of evidence that a large number of growth factors, such as transforming growth factor- $\beta$ (TGF- $\beta$ ), Wnt and epidermal growth factor (EGF), cytokines, such as tumor necrosis factor- $\alpha$, nuclear factor $-\kappa \mathrm{B}$ and Akt, transcription factors, such as Snail, Twist and Slug, and other markers are involved in the EMT process (48-50). However, the mechanism of the cooperative regulation between these factors in the EMT process remains unclear. EMT had been considered to be an important process in stimulating the metastasis of cervical cancer (51). Shostak et al (52) confirmed that HPV infection promoted EGF-mediated EMT in cervical cancer. In the current study, MMP1 may exert its activity through the PPAR pathway to alter the tumor microenvironment, thus inducing the EMT process by affecting the IL-6/STAT3 axis. Additionally, the stable expression of the active form of MMP1 was demonstrated to induce EMT by promoting the generation of TGF- $\beta$ (53), and certain EMT transcription factors, including Twist and Slug, have been reported to regulate MMP1 expression $(54,55)$. Relevant research is required to determine the detailed mechanism by which MMP1 promotes EMT during metastasis of cervical cancer.

The carcinogenic properties of MMP1 adversely affect the prognosis of patients with cervical cancer. Highly expressed MMP1 was significantly inversely associated with poor overall survival in a cohort of 306 patients based on TCGA data. MMP1 was an independent prognostic biomarker of cervical cancer. The clinical value of MMP1 has been reported in certain other types of human cancer $(15,31,45,56)$; however, the importance for cervical cancer remains unclear. Certain studies have noted the overexpression of MMP1 in cervical cancer $(47,57,58)$, while the current study suggested that MMP1 was an independent prognostic factor for cervical cancer. These findings will have a guiding role in future clinical work.

The research of the present study still has some shortcomings. Independent cervical cancer samples were not available to validate the function of MMP1, and also the relevant animal experiments have not been performed. Although MMP1 is highly expressed in cervical cancer from 
several independent databases, related MMP1 overexpression experiments were not conducted. The use of stable cell lines with MMP1 overexpression will be required for further research. The detailed mechanism by which MMP1 promotes cervical cancer metastasis required further study. These are the future research directions, some of which have already been performed.

In summary, the data of the current study indicated that the PPAR signaling pathway has important role during LN metastasis of cervical cancer, and that MMP1 in the PPAR signaling pathway acted as a key role in the regulation of tumor growth and LN metastasis via EMT process in cervical cancer. MMP1 was suggested to be a biomarker for LN metastasis and an independent prognostic factor in cervical cancer that requires further validation.

\section{Acknowledgements}

The authors thank Professor Xu Dakang (Monash University, Clayton, Australia) for advice on the manuscript and Dr Henry Clarke (Monash University) for work on the language of this manuscript.

\section{Funding}

No funding was received.

\section{Availability of data and materials}

The datasets used and/or analyzed during the current study are available from the corresponding author on reasonable request.

\section{Authors' contributions}

RT analyzed the bioinformatic data. RT and XFL performed the cell assays and were major contributors in writing the manuscript. YG, YL, PY and KZW gave advice on the experiments and writing of the manuscript. RT and KZW designed the study. All authors read and approved the final manuscript.

\section{Ethics approval and consent to participate}

Not applicable.

\section{Consent for publication}

Not applicable.

\section{Competing interests}

The authors declare that they have no competing interests.

\section{References}

1. Siegel RL, Miller KD and Jemal A: Cancer Statistics, 2017. CA Cancer J Clin 67: 7-30, 2017.

2. Munro A, Codde J, Spilsbury K, Steel N, Stewart CJ, Salfinger SG, Tan J, Mohan GR, Leung Y, Semmens JB, et al: Risk of persistent and recurrent cervical neoplasia following incidentally detected adenocarcinoma in situ. Am J Obstet Gynecol 216: 272.e1-272. e7, 2017.
3. Kim S-W, Chun M, Ryu H-S, Chang SJ, Kong TW, Lee EJ, Lee YH and Oh YT: Salvage radiotherapy with or without concurrent chemotherapy for pelvic recurrence after hysterectomy alone for early-stage uterine cervical cancer. Strahlenther Onkol 193: 534-542, 2017.

4. Li C, Liu W and Cheng Y: Prognostic significance of metastatic lymph node ratio in squamous cell carcinoma of the cervix. Onco Targets Ther 9: 3791-3797, 2016.

5. Wang XJ, Xiong Y, Ma ZB, Xia JC and Li YF: The expression and prognostic value of protein tyrosine kinase 6 in early-stage cervical squamous cell cancer. Chin J Cancer 35: 54, 2016.

6. Li J, Wu MF, Lu HW, Chen Q, Lin ZQ and Wang LJ: Pretreatment serum lactate dehydrogenase is an independent prognostic factor for patients receiving neoadjuvant chemotherapy for locally advanced cervical cancer. Cancer Med 5: 1863-1872, 2016.

7. Fleming ND, Frumovitz M, Schmeler KM, dos Reis R, Munsell MF, Eifel PJ, Soliman PT, Nick AM, Westin SN and Ramirez PT: Significance of lymph node ratio in defining risk category in node-positive early stage cervical cancer. Gynecol Oncol 136: 48-53, 2015.

8. Zhang WN, Li W, Wang XL, Hu Z, Zhu D, Ding WC, Liu D, Li KZ, Ma D and Wang H: CLDN1 expression in cervical cancer cells is related to tumor invasion and metastasis. Oncotarget 7: 87449-87461, 2016.

9. Chung IH, Wu TI, Liao CJ, Hu JY, Lin YH, Tai PJ, Lai CH and Lin KH: Overexpression of lipocalin 2 in human cervical cancer enhances tumor invasion. Oncotarget 7: 11113-11126, 2016.

10. Issemann I and Green S: Activation of a member of the steroid hormone receptor superfamily by peroxisome proliferators. Nature 347: 645-650, 1990.

11. Berger J and Moller DE: The mechanisms of action of PPARs. Annu Rev Med 53: 409-435, 2002.

12. Mandard S and Patsouris D: Nuclear control of the inflammatory response in mammals by peroxisome proliferator-activated receptors. PPAR Res 2013: 613864, 2013.

13. Xin X, Yang S, Kowalski J and Gerritsen ME: Peroxisome proliferator-activated receptor gamma ligands are potent inhibitors of angiogenesis in vitro and in vivo. J Biol Chem 274: 9116-9121, 1999.

14. Sato M: Peroxisome proliferator activated receptor ligands and angiogenesis. Nihon Rinsho 63: 603-608, 2005 (In Japanese).

15. Liu M, Hu Y, Zhang MF, Luo KJ, Xie XY, Wen J, Fu JH and Yang H: MMP1 promotes tumor growth and metastasis in esophageal squamous cell carcinoma. Cancer Lett 377: 97-104, 2016.

16. Ozden F, Saygin C, Uzunaslan D, Onal B, Durak H and Aki H: Expression of MMP-1,MMP-9 and TIMP-2 in prostate carcinoma and their influence on prognosis and survival. J Cancer Res Clin Oncol 139: 1373-1382, 2013.

17. Shin DH, Dier U, Melendez JA and Hempel N: Regulation of MMP-1 expression in response to hypoxia is dependent on the intracellular redox status of metastatic bladder cancer cells. Biochim Biophys Acta 1852: 2593-2602, 2015.

18. Cai QW, Li J, Li XQ, Wang JQ and Huang Y: Expression of STAT3, MMP-1 and TIMP-1 in gastric cancer and correlation with pathological features. Mol Med Rep 5: 1438-1442, 2012.

19. Juncker-Jensen A, Deryugina EI, Rimann I, Zajac E, Kupriyanova TA, Engelholm LH and Quigley JP: Tumor MMP-1 activates endothelial PAR1 to facilitate vascular intravasation and metastatic dissemination. Cancer Res 73: 4196-4211, 2013.

20. Anand M, Van Meter TE and Fillmore HL: Epidermal growth factor induces matrix metalloproteinase-1 (MMP-1) expression and invasion in glioma cell lines via the MAPK pathway. J Neurooncol 104: 679-687, 2011.

21. Scotto L, Narayan G, Nandula SV, Arias-Pulido H, Subramaniyam S, Schneider A, Kaufmann AM, Wright JD, Pothuri B, Mansukhani M, et al: Identification of copy number gain and overexpressed genes on chromosome arm 20q by an integrative genomic approach in cervical cancer: Potential role in progression. Genes Chromosomes Cancer 47: 755-765, 2008.

22. Robinson MD, McCarthy DJ and Smyth GK: edgeR: A Bioconductor package for differential expression analysis of digital gene expression data. Bioinformatics 26: 139-140, 2010.

23. McCarthy DJ, Chen Y and Smyth GK: Differential expression analysis of multifactor RNA-Seq experiments with respect to biological variation. Nucleic Acids Res 40: 4288-4297, 2012.

24. Huber W, Carey VJ, Gentleman R and Anders S: Orchestrating high-throughput genomic analysis with Bioconductor. Nat Methods 12: 115-121, 2015. 
25. Ritchie ME, Phipson B, Wu D, Hu Y, Law CW, Shi W and Smyth GK: limma powers differential expression analyses for RNA-sequencing and microarray studies. Nucleic Acids Res 43: e47, 2015 .

26. Mootha VK, Lindgren CM, Eriksson KF, Subramanian A, Sihag S, Lehar J, Puigserver P, Carlsson E, Ridderstråle M, Laurila E, et al: PGC-1alpha-responsive genes involved in oxidative phosphorylation are coordinately downregulated in human diabetes. Nat Genet 34: 267-273, 2003.

27. Subramanian A, Tamayo P, Mootha VK, Mukherjee S, Ebert BL, Gillette MA, Paulovich A, Pomeroy SL, Golub TR, Lander ES, et al: Gene set enrichment analysis: A knowledge-based approach for interpreting genome-wide expression profiles. Proc Natl Acad Sci USA 102: 15545-15550, 2005.

28. Aubrey BJ, Kelly GL, Kueh AJ, Brennan MS, O'Connor L, Milla L, Wilcox S, Tai L, Strasser A and Herold MJ: An inducible lentiviral guide RNA platform enables the identification of tumor-essential genes and tumor-promoting mutations in vivo. Cell Reports 10: 1422-1432, 2015.

29. Li X, Tian R, Gao H, Yang Y, Williams BRG, Gantier MP McMillan NAJ, Xu D, Hu Y and Gao Y: Identification of a histone family gene signature for predicting the prognosis of cervical cancer patients. Sci Rep 7: 16495, 2017.

30. Livak KJ and Schmittgen TD: Analysis of relative gene expression data using real-time quantitative PCR and the 2(-Delta Delta C(T)) Method. Methods 25: 402-408, 2001.

31. Michalik L, Desvergne B and Wahli W: Peroxisome-proliferatoractivated receptors and cancers: Complex stories. Nat Rev Cancer 4: 61-70, 2004

32. Cangemi A, Fanale D, Rinaldi G, Bazan V, Galvano A, Perez A, Barraco N, Massihnia D, Castiglia M, Vieni S, et al: Dietary restriction: Could it be considered as speed bump on tumor progression road? Tumour Biol 37: 7109-7118, 2016

33. O'Sullivan KE, Reynolds JV, O'Hanlon C, O'Sullivan JN and Lysaght J: Could signal transducer and activator of transcription 3 be a therapeutic target in obesity-related gastrointestinal malignancy? J Gastrointest Cancer 45: 1-11, 2014.

34. ZhuP, Goh YY, ChinHF, Kersten S and Tan NS: Angiopoietin-like 4 A decade of research. Biosci Rep 32: 211-219, 2012.

35. Pencik J, Schlederer M, Gruber W, Unger C, Walker SM, Chalaris A, Marié IJ, Hassler MR, Javaheri T, Aksoy O, et al: STAT3 regulated ARF expression suppresses prostate cancer metastasis. Nat Commun 6: 7736, 2015.

36. Zheng T, Hong X, Wang J, Pei T, Liang Y, Yin D, Song R, Song X, Lu Z, Qi S, et al: Gankyrin promotes tumor growth and metastasis through activation of IL-6/STAT3 signaling in human cholangiocarcinoma. Hepatology 59: 935-946, 2014.

37. Trombetta A, Maggiora M, Martinasso G, Cotogni P, Canuto RA and Muzio G: Arachidonic and docosahexaenoic acids reduce the growth of A549 human lung-tumor cells increasing lipid peroxidation and PPARs. Chem Biol Interact 165: 239-250, 2007.

38. Margeli A, Kouraklis G and Theocharis S: Peroxisome proliferator activated receptor-gamma (PPAR-gamma) ligands and angiogenesis. Angiogenesis 6: 165-169, 2003.

39. George A, Ranganathan K and Rao UK: Expression of MMP-1 in histopathological different grades of oral squamous cell carcinoma and in normal buccal mucosa - an immunohistochemical study. Cancer Biomark 7: 275-283, 2010.

40. Langenskiöld M, Ivarsson ML, Holmdahl L, Falk P, KåbjörnGustafsson C and Angenete E: Intestinal mucosal MMP-1 - a prognostic factor in colon cancer. Scand J Gastroenterol 48 . $563-569,2013$

41. Hua H, Li M, Luo T, Yin Y and Jiang Y: Matrix metalloproteinases in tumorigenesis: An evolving paradigm. Cell Mol Life Sci 68: 3853-3868, 2011.

42. Zhang Z, Tao D, Zhang P, Liu X, Zhang Y, Cheng J, Yan H, Liu L and Jiang H: Hyaluronan synthase 2 expressed by cancerassociated fibroblasts promotes oral cancer invasion. J Exp Clin Cancer Res 35: 181, 2016.
43. Panagopoulos V, Leach DA, Zinonos I, Ponomarev V, Licari G, Liapis V, Ingman WV, Anderson P, DeNichilo MO and Evdokiou A: Inflammatory peroxidases promote breast cancer progression in mice via regulation of the tumour microenvironment. Int J Oncol 50: 1191-1200, 2017.

44. Kuasne H, Barros-Filho MC, Busso-Lopes A, Marchi FA, Pinheiro M, Muñoz JJ, Scapulatempo-Neto C, Faria EF, Guimarães GC, Lopes A, et al: Integrative miRNA and mRNA analysis in penile carcinomas reveals markers and pathways with potential clinical impact. Oncotarget 8: 15294-15306, 2017.

45. Yokoi A, Yoshioka Y, Yamamoto Y, Ishikawa M, Ikeda SI, Kato T, Kiyono T, Takeshita F, Kajiyama H, Kikkawa F, et al: Malignant extracellular vesicles carrying MMP1 mRNA facilitate peritoneal dissemination in ovarian cancer. Nat Commun 8: 14470 , 2017.

46. Ismail TM, Bennett D, Platt-Higgins AM, Al-Medhity M, Barraclough R and Rudland PS: S100A4 elevation empowers expression of metastasis effector molecules in human breast cancer. Cancer Res 77: 780-789, 2017.

47. Sun N-x, Zhao Q, Ye C, Ma Y and Li W: Role of matrix metalloproteinase-1 (MMP-1)/protease-activated receptor-1 (PAR-1) signaling pathway in the cervical cancer invasion. J Reprod Contracept 25: 18-25, 2014.

48. Thiery JP, Acloque H, Huang RY and Nieto MA: Epithelialmesenchymal transitions in development and disease. Cell 139: 871-890, 2009.

49. Yang L, Han S and Sun Y: An IL6-STAT3 loop mediates resistance to PI3K inhibitors by inducing epithelial-mesenchymal transition and cancer stem cell expansion in human breast cancer cells. Biochem Biophys Res Commun 453: 582-587, 2014

50. Tse JC and Kalluri R: Mechanisms of metastasis: Epithelialto-mesenchymal transition and contribution of tumor microenvironment. J Cell Biochem 101: 816-829, 2007.

51. Huang L, Huang Z, Fan Y, He L, Ye M, Shi K, Ji B, Huang J, Wang Y and Li Q: FOXC1 promotes proliferation and epithelialmesenchymal transition in cervical carcinoma through the PI3K-AKT signal pathway. Am J Transl Res 9: 1297-1306, 2017.

52. Shostak K, Zhang X, Hubert P, Göktuna SI, Jiang Z, Klevernic I, Hildebrand J, Roncarati P, Hennuy B, Ladang A, et al: NF-kBinduced KIAA1199 promotes survival through EGFR signalling. Nat Commun 5: 5232, 2014.

53. Iida J and McCarthy JB: Expression of collagenase-1 (MMP-1) promotes melanoma growth through the generation of active transforming growth factor-beta. Melanoma Res 17: 205-213, 2007.

54. Weiss MB, Abel EV, Mayberry MM, Basile KJ, Berger AC and Aplin AE: TWIST1 is an ERK1/2 effector that promotes invasion and regulates MMP-1 expression in human melanoma cells. Cancer Res 72: 6382-6392, 2012.

55. Shen CJ, Kuo YL, Chen CC, Chen MJ and Cheng YM: MMP1 expression is activated by Slug and enhances multi-drug resistance (MDR) in breast cancer. PLoS One 12: e0174487, 2017.

56. Mueller E, Sarraf P, Tontonoz P, Evans RM, Martin KJ, Zhang M, Fletcher C, Singer S and Spiegelman BM: Terminal differentiation of human breast cancer through PPAR gamma. Mol Cell 1: 465-470, 1998

57. Zhang YX and Zhao YL: Pathogenic network analysis predicts candidate genes for cervical cancer. Comput Math Methods Med 2016: 3186051, 2016

58. Rajkumar T, Sabitha K, Vijayalakshmi N, Shirley S, Bose MV, Gopal G and Selvaluxmy G: Identification and validation of genes involved in cervical tumourigenesis. BMC Cancer 11: 80, 2011.

This work is licensed under a Creative Commons Attribution 4.0 International (CC BY 4.0) License. 